\title{
Cross-section measurements relevant for the astrophysical $p$ process at the University of Cologne
}

\author{
Felix Heim ${ }^{1, *}$, Martin Müller ${ }^{1}$, Philipp Scholz ${ }^{2}$, Svenja Wilden ${ }^{1}$, and Andreas Zilges ${ }^{1}$ \\ ${ }^{1}$ University of Cologne, Institute for Nuclear Physics, Germany \\ ${ }^{2}$ Department of Physics, University of Notre Dame, Indiana 46556-5670, USA
}

\begin{abstract}
The astrophysical $p$ process unites all processes that have been introduced to explain the abundance of a group of 30 to 35 neutron-deficient nuclei which are referred to as $p$ nuclei. In general, these $p$ processes include large networks of nuclear reactions and a complete understanding of the individual reaction rates is required to describe the abundance of the $p$ nuclei qualitatively and quantitatively. In many cases the involved nuclear reactions are not accessible in the laboratory, either due to their low cross sections or because they involve unstable or exotic isotopes. For those purposes, the motivation of crosssection measurements performed at the University of Cologne is twofold: First, experimentally constrained reaction rates are of direct relevance for nucleosynthesis network calculations. And second, experimental cross-section values are required to test existing theoretical descriptions and to improve their predictive power. In this work, we present the experimental setups and methods that are used to measure nuclear cross-sections at very low sensitivities and we show a detailed overview of proton- and $\alpha$-induced reactions that have been measured in Cologne in the last decade.
\end{abstract}

\section{Introduction}

The majority of stable isotopes is created by neutron-capture processes within various astrophysical scenarios $[1,2]$. However, a small group of nuclei on the neutron-deficient side of the valley of stability - the $p$ nuclei - demands for a different kind of nucleosynthesis mechanism $[3,4]$. The $\gamma$ process has been introduced for that purpose which represents a huge network of photodisintegration reactions which act on pre-produced nuclei near the valley of stability. Since the $\gamma$ process is a huge network of thousands of nuclear reactions a large database of experimentally constrained cross sections is necessary to model the abundance flow within the respective astrophysical scenario. Unfortunately, in many cases one has to rely on theoretical predictions and extrapolations because no experimental data are available within the astrophysical relevant energy region. For those reasons, the improvement of the sensitivity limit of experimental setups used for astrophysical cross-section measurements is of high importance. In addition, testing the predictive power of theoretical descriptions which are used to predict cross-section data in regions that are not accessible in the laboratory is one of the key motivations for cross-section experiments relevant for the $p$-process nucleosynthesis. In this contribution, different experimental methods and setups are briefly introduced that

\footnotetext{
*e-mail: fheim@ikp.uni-koeln.de
} 

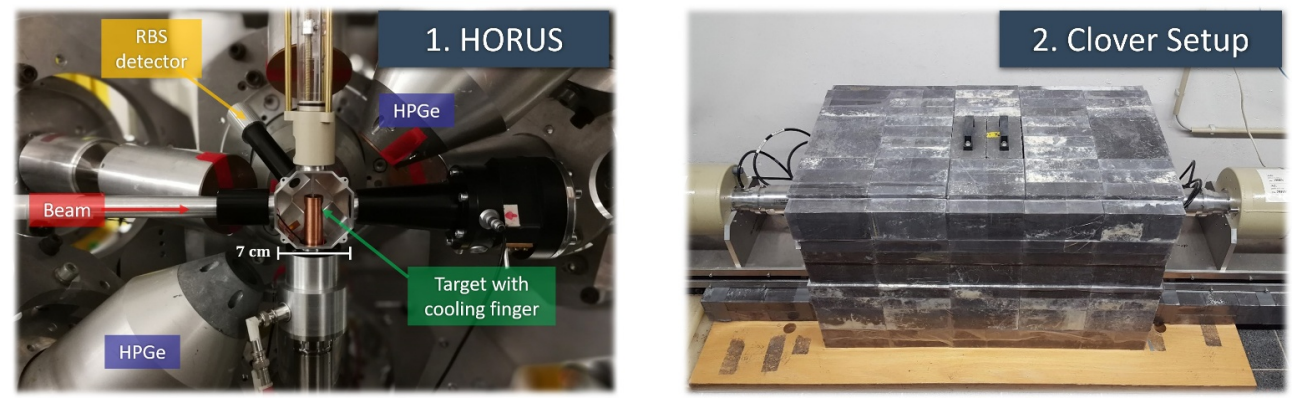

Figure 1. Left side: The $\gamma$-ray spectrometer HORUS and the target chamber dedicated for nuclear astrophysics [5]. The setup is used for in-beam cross-section measurements or for the irradiation of targets which can be transported to the dedicated offline counting setup (right side) for a subsequent analysis. The counting setup consists of two clover-type detectors which are shielded by lead and copper.

are used at the University of Cologne for cross-section measurements. In Sec. 3, an overview is given over proton- and $\alpha$-induced reactions that have been measured in Cologne over the last decade.

\section{Experimental setups and methods for cross-section measurements in Cologne}

In general, there are two main techniques to measure the cross-section of a nuclear reaction in the laboratory. Both methods are based on the idea to count the number of nuclear reactions and normalize them to the number of incoming projectiles and target nuclei (see. Eq. 1).

$$
\sigma=\frac{N_{\text {Reactions }}}{N_{P} \cdot N_{T}}
$$

The idea of the first method is to measure the prompt emission of particles emitted by the reaction product. In the case of emitted $\gamma$-radiation, this technique is called in-beam $\gamma$-ray spectroscopy. The method and its applicability is presented in detail in Ref. [5]. The second type of methods can be applied if the produced nuclei are radioactive and decay via the emission of any kind of radiation, e.g., via $\gamma$-emission or $\alpha$-emission. Via measuring the produced activity of the reaction products the number of produced nuclei during irradiation and hence, the nuclear cross section $\sigma$ can be derived. This method is therefore called activation method [6].

\subsection{In-beam $\gamma$-ray spectroscopy}

At the University of Cologne a dedicated target chamber is available that has been explicitly tailored to the needs of astrophysical cross-section measurements. The chamber is mounted within the $\gamma$-ray spectrometer HoRus and is very compact in its dimension (about $7 \mathrm{~cm}$ in length). That allows to move all detectors very close to the target position and yields a full-energy peak efficiency of about $3 \%$ at $E_{\gamma}=1.3 \mathrm{MeV}$. The target chamber, the $\gamma$-ray spectrometer and the experimental methods are explained in detail in Ref. [5] and the chamber is shown on the left side in Fig. 1. During the last decade, a multitude of proton- and 
$\alpha$-induced reactions has been measured in Cologne by means of in-beam $\gamma$-ray spectroscopy, see Fig. 2 or Tab. 1. The obtained cross sections have not only been used to be compared to existing theoretical models but the detection of the prompt $\gamma$-rays also allowed to study more fundamental nuclear properties such as the $\gamma$-ray strength function or the nuclear level density, as described in Refs. [7, 8].

\subsection{Activation experiments}

If the reaction product is unstable and decays via the emission of particles or $\gamma$ rays, the activated material can be transported to a dedicated counting setup after the end of irradiation. At the University of Cologne a low-background counting setup is available which consists of two HPGe clover detectors which are shielded by lead and copper. The setup has a fullenergy peak efficiency of about $5.3 \%$ at a $\gamma$-ray energy of $E_{\gamma}=1.3 \mathrm{MeV}$ [9] and is shown on the right side of Fig. 1. Using the $\gamma$-ray counting setup, various proton- and $\alpha$-induced reactions have been investigated in Cologne within the last years. In addition to single-target irradiation, we performed stacked-target experiments for the ${ }^{170,172} \mathrm{Yb}(\alpha, \mathrm{n}){ }^{173,175} \mathrm{Hf}$ reactions using water-cooled stacks which consist of four targets each [10].

Apart from $\gamma$-ray counting experiments, we have been able to measure the astrophysical relevant ${ }^{144} \operatorname{Sm}(\alpha, \gamma){ }^{148} \mathrm{Gd}$ reaction at energies close to the Gamow window. As ${ }^{148} \mathrm{Gd}$ does not emit any $\gamma$ rays within its $\alpha$-decay, we performed an $\alpha$-counting experiment [11].

\section{Overview of investigated reactions}

Over the last decade, a multitude of nuclear reactions involving heavy nuclei has been investigated by means of different techniques at the University of Cologne. The derived crosssection data are inevitable for nucleosynthesis network calculations, in particular they are relevant for $p$-process nucleosynthesis. Additionally, the experimental data are required to benchmark theoretical models that are used to predict reaction rate data in nuclear regions that are not (yet) accessible via experiments and to put the models on a firm basis. The explicit nuclear reactions investigated in Cologne are listed in Tab. 1 and depicted in Fig. 2. In the future, detailed investigations of nuclear reactions relevant for nuclear astrophysics will be continued in Cologne, aiming at extending the available database of measured cross sections.

Table 1. List of proton- and $\alpha$-induced reaction cross sections relevant for nuclear astrophysics measured at the University of Cologne over the last decade.

\begin{tabular}{|c|c|c|c|}
\hline$(p, \gamma)$ & $(p, n)$ & $(\alpha, \gamma)$ & $(\alpha, n)$ \\
\hline${ }^{85} \mathrm{Rb}(p, \gamma){ }^{86} \mathrm{Sr}[12]$ & $\left.{ }^{96} \mathrm{Mo}(p, n)\right)^{96 m, g} \mathrm{Tc}[9]$ & ${ }^{107} \mathrm{Ag}(\alpha, \gamma){ }^{111} \operatorname{In}[20]$ & ${ }^{107} \operatorname{Ag}(\alpha, n){ }^{110} \operatorname{In}[20]$ \\
\hline${ }^{87} \mathrm{Rb}(p, \gamma){ }^{88} \mathrm{Sr}[12]$ & & ${ }^{108} \mathrm{Cd}(\alpha, \gamma){ }^{112} \mathrm{Sn}[21]$ & ${ }^{108} \mathrm{Cd}(\alpha, n){ }^{111} \mathrm{Sn}[21]$ \\
\hline${ }^{89} \mathrm{Y}(p, \gamma)^{90} \mathrm{Zr}[5,8,13]$ & & ${ }^{112} \mathrm{Sn}(\alpha, \gamma){ }^{116} \mathrm{Te}[24]$ & ${ }^{141} \operatorname{Pr}(\alpha, n){ }^{144} \operatorname{Pm}[22]$ \\
\hline${ }^{92} \operatorname{Mo}(p, \gamma){ }^{93} \mathrm{Tc}[14]$ & & ${ }^{144} \mathrm{Sm}(\alpha, \gamma){ }^{148} \mathrm{Gd}[11]$ & ${ }^{168} \mathrm{Yb}(\alpha, n){ }^{171} \mathrm{Hf}[23]$ \\
\hline${ }^{93} \mathrm{Nb}(p, \gamma){ }^{94} \mathrm{Mo}[7,15]$ & & ${ }^{168} \mathrm{Yb}(\alpha, \gamma){ }^{172} \mathrm{Hf}[23]$ & ${ }^{170} \mathrm{Yb}(\alpha, n){ }^{173} \mathrm{Hf}[10]$ \\
\hline${ }^{96} \mathrm{Mo}(p, \gamma){ }^{97} \mathrm{Tc}[16]$ & & & ${ }^{172} \mathrm{Yb}(\alpha, n){ }^{175} \mathrm{Hf}[10]$ \\
\hline${ }^{107} \mathrm{Ag}(p, \gamma){ }^{108} \mathrm{Cd}[17]$ & & & \\
\hline${ }^{109} \mathrm{Ag}(p, \gamma){ }^{110} \mathrm{Cd}[18]$ & & & \\
\hline${ }^{130} \mathrm{Ba}(p, \gamma){ }^{131} \mathrm{La}[19]$ & & & \\
\hline
\end{tabular}




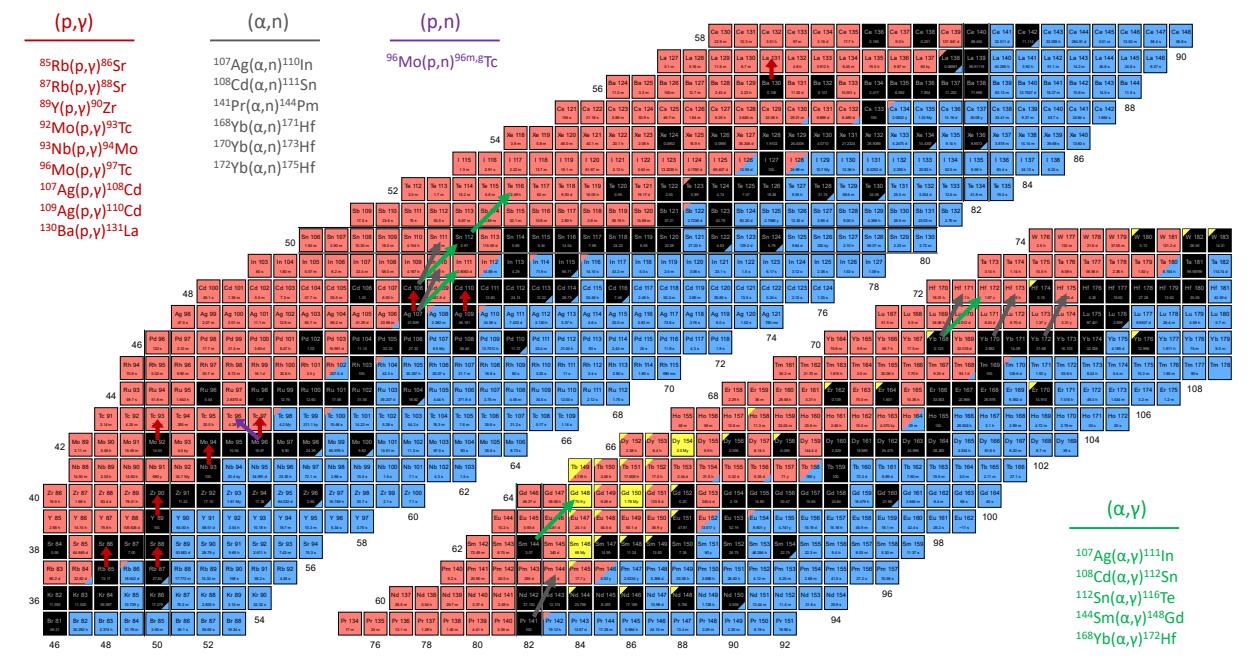

Figure 2. Illustration of proton- and $\alpha$-induced reaction cross sections relevant for nuclear astrophysics measured at the University of Cologne over the last decade.

\section{References}

[1] F. Käppeler et al., Rev. Mod. Phys. 83157 (2011)

[2] J. J. Cowan et al., Rev. Mod. Phys. 93 015002(2021)

[3] M. Arnould and S. Goriely, Phys. Rep. 3841 (2003)

[4] T. Rauscher et al., Rep. Prog. Phys. 7676 (2013)

[5] F. Heim et al., Nucl. Instr. Meth. Phys. Res. A 966163854 (2020)

[6] Gy. Gyürky et al., Eur. Phys. J. A 5541 (2019)

[7] F. Heim et al., Phys. Rev. C 103025805 (2021)

[8] L. Netterdon et al., Phys. Lett. B 744358 (2015)

[9] F. Heim et al., Phys. Rev. C 103054613 (2021)

[10] M. Müller et al., to be published

[11] P. Scholz et al., Phys. Rev. C 102045811 (2020)

[12] S. Wilden et al., to be published

[13] L. Netterdon et al., Nucl. Instr. Meth. Phys. Res. A 75494 (2014)

[14] J. Mayer et al., Phys. Rev. C 93045809 (2016)

[15] F. Heim et al., Phys. Rev. C 101035807 (2020)

[16] F. Heim et al., to be published

[17] F. Heim et al., Phys. Rev. C 101035805 (2020)

[18] F. Heim et al., Phys. Rev. C 103055803 (2021)

[19] L. Netterdon et al., Phys. Rev. C 90035806 (2014)

[20] Y. Wang et al., to be published

[21] P. Scholz et al., Phys. Lett. B 761247 (2016)

[22] A. Sauerwein et al., Phys. Rev. C 84045808 (2011)

[23] L. Netterdon et al., Nucl. Phys. A 916149 (2013)

[24] L. Netterdon et al., Phys. Rev. C 91035801 (2015) 